\title{
Comparison of the clinical manifestations and cement distribution after percutaneous vertebroplasty between thoracolumbar Kümmell's disease and OVCFs
}

\section{Cheng-Liang Wang}

CR \& WISCO General Hospital

Ji-Bin Chen ( $\sim 260636672 @ q q . c o m$ )

Wuhan Hanyang Hospital

Te Li

General Hospital of Central Theater Command

\section{Research article}

Keywords: Kümmell's disease, Osteoporosis; Vertebral compression fracture; Percutaneous vertebroplasty

Posted Date: July 14th, 2020

DOI: https://doi.org/10.21203/rs.3.rs-39692/v1

License: (c) (i) This work is licensed under a Creative Commons Attribution 4.0 International License.

Read Full License 


\section{Abstract}

Background Kümmell's disease is a chronic, non-healing osteoporotic vertebral compression fractures (OVCFs), which is associated with ischemic necrosis and vertebral pseudarthrosis. The purpose of this study was to compare the therapeutic effect and distribution characteristics of bone cement in the percutaneous vertebroplasty (PVP) treatment between thoracolumbar Kümmell's diseases and OVCFs.

Methods: A total of 70 patients with Kümmell 's disease (K group) or OVCFs (O group) who underwent PVP surgery from February 2016 to February 2018 were included in this study. The follow-up time was 24-48 months. The course of disease was significantly longer in the $\mathrm{K}$ group than in the 0 group $(P=0.000)$. Most cases in the $\mathrm{K}$ group had no history of trauma, while most cases in the 0 group had a history of falls $(P=0.001)$. The vertebral compression rate and degree of osteoporosis were more serious in the $\mathrm{K}$ group than in the $\mathrm{O}$ group ( $P<0.05$, respectively). Distribution pattern, volume and leakage rate of bone cement, operation time, VAS score, ODI, correction rates of vertebral compression and kyphosis, refracture rate of adjacent vertebrae between the two groups were compared to assess clinical effect.

Results: The amount of bone cement injected was significantly higher in the $\mathrm{K}$ group than in the 0 group $(P=0.025)$. The cement distribution pattern of local solid lump was dominant in the $\mathrm{K}$ group $(65.71 \%)$, while intercalation with trabeculae was dominant in the 0 group $(74.29 \%)(P<0.001)$. VAS and ODI were significantly lower both in the two groups at the 1st day, 1st year and 2nd years after surgery than before surgery (all $P<0.05$ ), but significantly lower in the $\mathrm{K}$ group than in the $\mathrm{O}$ group at each time point after surgery (all $P<0.05$ ). The correction rates of kyphosis and vertebral compression in both groups were significantly improved ( $P<0.05$, respectively) and gradually lost with time $(P<0.05$, respectively). The correction rates of kyphosis and vertebral compression were significantly higher in the $\mathrm{K}$ group than in the 0 group at each time point after surgery (all $P<0.05$ ).

Conclusion: Kümmell's disease has a higher degree of disease progression and osteoporosis than OVCFs. PVP can better restore partial vertebral height and correct kyphosis in the treatment of Kümmell's disease, while can better alleviate pain and improve ODI in the treatment of OVCFs.

\section{Background}

Kümmell's disease is a chronic, non-healing osteoporotic vertebral compression fractures (OVCFs), which is associated with the ischemic necrosis of vertebral body after fracture and vertebral pseudarthrosis [1, 2]. Due to the continuous collapse of vertebral body after minor trauma, clinical manifestations are often intractable low back pain, and even the symptoms of spinal nerve function damage [3]. Thoracolumbar vertebrae is the most common site of its occurrence. The characteristic performance of imaging findings are intravertebral vacuum cleft (IVC) and intravertebral vacuum phenomenon (IVP) $[4,5]$.

Percutaneous vertebroplasty (PVP) is often used in OVCFs patients because of its minimally invasive approach, stable vertebra and rapid pain relief [6]. Besides, PVP can partially restore vertebral height and correct kyphosis. PVP surgery can reduce the risk of vertebral wedge change and nerve injury in 
Kümmell's disease, so early PVP surgery should be performed [7]. However, between OVCFs and Kümmell's disease, the clinical efficacy, clinical characteristics, and complications of PVP treatment are still uncertain [8].

Therefore, in order to investigate the clinical efficacy, clinical characteristics, and complications of PVP treatment between OVCFs and Kümmell's disease, we conducted a retrospective study in our hospitals from February 2016 to February 2018 to analyze the clinical data between single-thoracolumbar Kümmell's disease and OVCFs for providing references of clinical practice and research.

\section{Materials And Methods}

\section{Patients}

This study is a retrospective study. Inclusion criteria were as follows: (1) Patients suffered single thoracolumbar either OVCFs or Kümmell's disease (2) The location of the "pain vertebrae" of physical examination was consistent with the fractured site of imaging examination. (3) Bone mineral density $\triangle B M D \llbracket$ of dual-energy $X$-ray examination was less than -2.5 (T value); (4) Follow-up time exceeded 2 years; (5) The patients used anti-osteoporosis drugs and calcium supplementation after surgery. Exclusion criteria were as follows: (1) Surgical contraindication was found, such as severe cardiopulmonary dysfunction, coagulation dysfunction, and local or systemic infection; (2) Symptoms of nerve root or spinal cord compression were observed; (3) Pathological fractures, other than osteoporosis, were found; (4) Adjacent vertebral bodies were observed with new OVCFs or obvious compression wedge deformation.

\section{General information}

A total of 70 cases from February 2016 to February 2018 met the selection criteria in the study, of which 35 cases of Kümmell's disease (K group) and 35 cases of OVCFs (O group) received PVP surgery (Figure 1). This study was approved by the ethics committees of our hospitals. All clinical data and pictures of the patients were obtained written informed consent from the patients or their families.

The two groups were followed up for 24-48 months. There were not significant differences in gender, age, injury segment, preoperative VAS and Oswestry dysfunction index (ODI) between the two groups (all $P>0.05)$. The course of disease was significantly longer in the $\mathrm{K}$ group than in the 0 group $(P=0.000)$. Most cases in the $\mathrm{K}$ group had no history of trauma, while most cases in the $\mathrm{O}$ group had a history of falls $(P=0.001)$. The vertebral compression rate and the degree of osteoporosis of the $\mathrm{K}$ group were more serious than those of the 0 group $(P<0.05$, respectively). (Table 1$)$

\section{Surgical technique}

The PVP procedures both groups were performed by the same group of surgeons and followed the principle of consistency. the prone position were placed for these patients to maintain posterior extension of spine for completing postural reduction. After local anesthesia, C-arm X-ray machine fluoroscopy was 
used to guide bilateral pedicle punctures. The working cannula was placed to reach the anterior $1 / 3$ of the vertebral body or was adjacent to the IVC region. Polymethylmethacrylate (Tecres S.P.A., Verona, Italy) was injected into the vertebral body and the IVC region using two $3.5 \mathrm{~mm}$ lateral open bone cement injectors (Shanghai Kinetic Medical Co., Ltd., Shanghai, China) until cement distributions were well dispersed and satisfactory.

\section{Postoperative management}

After the surgery, these patients should rest in the supine position for 8 to 12 hours. These patients were mainly bed rest in the first 1-2 months. After 1-2 months of the surgery, low back and lumbar support braces were used to get out of bed. Anti-osteoporosis drugs, such as bisphosphonates, vitamin D and calcium tablets, were given for anti-osteoporosis treatment.

\section{Outcome measurements}

The distribution pattern, leakage rate and injected amount of bone cement, operation time, VAS score of low back [9], ODI [10] and the incidence of new adjacent vertebral fracture in 2 years were compared between the two groups.

Anteroposterior and lateral X-ray images were taken before and after the surgery to measure the height of the anterior edge and kyphosis angle (Cobb's angle) of the injured vertebral body. The above indicators were used to calculate the rate of vertebral compression, correction rate of vertebral compression, namely (rate of vertebral compression preoperatively - rate of vertebral compression postoperatively) / rate of vertebral compression preoperatively $\times \%$, and correction rate of kyphosis [11].

\section{Statistical analysis}

Statistical analysis was conducted by SPSS 19.0 statistical software (IBM Corp., Armonk, NY, USA). Measurement data were expressed as mean \pm standard deviation. The homogeneity of variance was tested by Levene test. Independent sample $t$ test was used for inter-group comparison. One-way ANOVA was used for comparison between different time points between groups. Repeated measurement ANOVA was used for intra-group comparison between different time points. The counting data were tested with $\chi^{2}$ test. $P<0.05$ was considered statistically difference.

\section{Results}

There were not statistical differences in follow-up time, operation time, intraoperative blood loss, or fluoroscopy times between the two groups (all $P>0.05$ ). The amount of bone cement injected was significantly higher in the $\mathrm{K}$ group than in the 0 group $(P=0.025)$. In the $\mathrm{K}$ group, 23 cases $(65.71 \%)$ were local solid lump distribution of bone cement (Figure 2a), and 12 cases (34.29\%) were embedded trabeculae of bone cement distribution pattern. In the 0 group, 9 cases $(25.71 \%)$ were local solid lump of distribution pattern of bone cement and 26 cases (74.29\%) were embedded trabeculae of bone cement 
distribution pattern (Figure 2b). The comparison of bone cement distribution pattern between the two groups was significantly different $(P<0.001)$.

Bone cement leakage occurred in 5 patients (14.29\%) in the K group, including 2 cases of leakage to the anterior wall, 1 case of upper intervertebral space, 1 case of lower intervertebral space and 1 case of lateral wall. In the 0 group, bone cement leakage occurred in 3 cases (8.57\%), including 1 case of lower intervertebral space, 1 case of upper intervertebral space and 1 case of lateral wall. However, none of these patients both the two groups had any related clinical symptoms. There was not significant difference in the incidence of cement leakage between the two groups $(P>0.05)$.(Table 2$)$

VAS and ODI were significantly lower in the two groups at the 1st day, 1 st year and 2nd year after surgery than before surgery (all $P<0.05$ ), but significantly lower in the $\mathrm{K}$ group than in the 0 group at each time point after surgery (all $P<0.05)$. During the two years follow-up, 6 patients $(17.14 \%)$ in the $\mathrm{K}$ group and 4 patients $(11.43 \%)$ in the 0 group had adjacent vertebral fractures $(P=0.243)$.(Table 2$)$

The correction rates of kyphosis and vertebral compression were significantly improved in both groups $(P<0.05$, respectively), but gradually decreased significantly with time $(P<0.05$, respectively). The correction rates of kyphosis and vertebral compression were significantly higher in the $\mathrm{K}$ group than in the 0 group at each time point after surgery (all $P<0.05$ ). (Table 3 )

\section{Discussion}

Kümmell's disease is a rare and special type of OVCFs. After minor trauma, vertebral collapse and kyphosis are gradually aggravated, which is related to vertebral ischemia, necrosis and pseudarthrosis $[12,13]$. Patients often suffer intractable lumbar and back pain, and even nerve injury. Imaging examination shows IVC or localized fluid filling in the vertebral body [14]. In this study, the course of Kümmell's disease was significantly longer than the course of OVCFs. Kümmell's disease mostly has not obvious trauma, but OVCFs mostly has a history of trauma. The degree of osteoporosis and vertebral compression rate were more serious in Kümmell's disease than in OVCFs.

Kümmell's disease is the severe stage of OVCFs. The goal of the treatment is to stop the progression of vertebral necrosis and rebuild spinal stability. Conservative treatment can make patients enter a vicious circle of ischemia and necrosis, while open surgery is not the best choice due to the large injury and slow recovery of elderly patients [15]. PVP technology has become the preferred method with the advantages of minimum injury and quick recovery. After the fissures of the fractured vertebral body filled with bone cement, the abnormal activity of the vertebral body was eliminated and the fractured vertebrae was stabilized, which was an important reason for pain relief [16]. Besides, the height of the vertebral body was partially restored and kyphosis deformity was partially corrected [16-24]. The ODI and VAS after PVP surgery both in Kümmell's disease and OVCFs groups were significantly decreased, but the mean scores were higher in the Kümmell's disease group than in the OVCFs groups [25]. Our study is consistent with the above studies, and the ODI and VAS in both groups can be maintained until the last follow-up. 
The amount of bone cement injected was not significantly correlated with pain relief, even $1.5 \mathrm{ml}$ of bone cement injected into each vertebral body can obtain satisfactory relief of the pain [26, 27]. Approximately $2 \mathrm{ml}$ of bone cement injected into the each vertebral body can restore the strength of the vertebral body, and Approximately $4 \mathrm{ml}$ of bone cement can restore the stiffness of vertebral body [27]. In this study, the average amount of bone cement injected was significantly higher in the $\mathrm{K}$ group $(4.2 \pm 1.15 \mathrm{ml})$ than in the 0 group $(3.6 \pm 1.35 \mathrm{ml})$. Both groups met the requirement of restoring vertebral strength, and the $\mathrm{K}$ group met the requirement of restoring vertebral stiffness. Bone cement can be injected in the $\mathrm{K}$ group higher than in the $\mathrm{O}$ group, which may be related to the presence of IVC and pseudarthrosis in vertebral bodies. So, after spine extension reduction, a larger reduction cavity can be obtained for bone cement injection.

When the spine flexes and bends, due to the presence of IVC and pseudarthrosis of Kümmell's disease, the injured vertebral body can be extended and stretched to widen the cracks. So, patients of Kümmell's disease can achieve spontaneous reduction in the posterior extension position without further balloon expansion reduction [28, 29]. Besides, Heo et al. [34] reported that excessive reduction of injured vertebral body will accelerate the process of vertebral ischemic necrosis, resulting in severe re-collapse. Therefore, considering cost and benefit, PVP has clinical superiority over PKP [11]. Due to the lack of IVC and vertebral pseudarthrosis, OVCFs is less effective than Kummell's disease in the reduction of posterior extension of the spine. So, our study found that the correction rates of vertebral compression and kyphosis after PVP were significantly corrected in both groups, but significant correction was achieved at each time point in the $\mathrm{K}$ group compared with the $\mathrm{O}$ group, further confirming the view that spontaneous vertebral reduction of Kümmell's disease can occur in the position of posterior extension.

The correction rates of vertebral compression rate and kyphosis both in the two groups gradually lost with time, which was consistent with previous findings [30, 31]. In the vertebral intensive treatment of OVCFs, bone cement in the PVP group was mainly embedded in cancellous bone, while bone cement in the PKP group was mainly distributed in clumps, so stress blocking is more likely to occur after PKP surgery, resulting in recollapse [32]. In this study, local solid lump distribution pattern of bone cement was dominant in the $\mathrm{K}$ group (65.71\%), while embedded trabeculae distribution pattern was dominant in the 0 group $(74.29 \%)$. In the two groups, two years after the surgery, re-collapse and intervertebral height loss occurred in the enhanced vertebra, but the loss in the $\mathrm{K}$ group was more significant than that in the 0 group. As a "reservoir" of IVC region, bone cement in the IVC region was filled in the form of solid clumps. The limited bone cement clumps cannot connect with the adjacent upper and lower endplates to strengthen cancellous bone of the vertebral body, thus failing to support the normal physiological stress and being prone to recollapse [33]. In addition, Kümmell's disease has a higher degree of disease process and osteoporosis than OVCFs, so the probability of recurrence fracture and recollapse in the K group is higher than that in the 0 group.

The most common complications after PVP are bone cement leakage and new adjacent vertebral fractures [34, 35]. Krauss et al. [36] reported that the rate of bone cement leakage was $18.2 \%$ in the treatment of Kümmell's disease by PVP. The reported rate of cement leakage of PVP in the treatment of 
OVCFs is about $10.56 \%-30 \%$ [37]. Our study found that the leakage rate of bone cement in the $\mathrm{K}$ and $\mathrm{O}$ groups were $14.29 \%$ and $8.57 \%$, respectively, which may be related to accurate surgical approach measurement preoperatively, careful surgical operation intraoperatively and not pursuing the maximum amount of bone cement. There was not significant difference in cement leakage rate between the two groups. $14.1 \%$ of OVCFs in the early PVP group and $39.1 \%$ in the late PVP group experienced a new adjacent vertebral fracture during the first year after PVP surgery [38]. In this study, the incidence of new adjacent vertebral fractures of the $\mathrm{K}$ group and $\mathrm{O}$ group were $17.14 \%$ and $11.43 \%$, respectively. There was not statistical difference between the two groups.

This study has some limitations. Firstly, The nature of the retrospective study itself cannot be avoided. Second, the number of cases was small, which needs to be further analyzed and clarified by expanding sample size. Moreover, there was a lack of time biomechanical study on cement distribution and IVC size in vertebral body to support the results. Lastly, patients and data collection researchers were not completely randomized double-blind. So, the current findings require further validation in multicenter clinical trials.

\section{Conclusion}

Kümmell's disease has a higher degree of disease progression and osteoporosis than OVCFs. PVP can better restore partial vertebral height and correct kyphosis in the treatment of Kümmell's disease, while can better alleviate pain and improve ODI in the treatment of OVCFs.

\section{Declarations}

\section{Ethics approval and consent to participate}

This study were approved by the institutional review boards/Ethics Committees of Wuhan Hanyang Hospital, CR \& WISCO General Hospital and General Hospital of Central Theater Command, and was conducted in compliance with the ethical principles of the Helsinki Declaration of 1975. Written informed consent was obtained from the patients or their family members.

\section{Consent for publication}

Not applicable.

\section{Availability of data and materials}

The datasets used and/or analysed during the current study are available from the corresponding author on reasonable request.

\section{Competing interests}

No potential conflict of interest relevant to this article was reported. 


\section{Funding}

There is no funding for the current study.

\section{Authors' contributions}

All authors made substantive intellectual contributions in this study to qualify as authors. C JZ and L T designed this study. W CL participated in collecting and analyzing raw materials. An initial draft of the manuscript was written by W CL. C JZ and L T re-drafted parts of the manuscript and provided helpful advice on the final revision. All authors were involved in writing the manuscript. All authors read and approved the final manuscript.

\section{Acknowledgements}

We are grateful to the surgeons, anesthesiologist, and radiologist, without whom this study would not have been possible: Dr Jing-Wu Chen, Kun Liu and Da-Zhi Wang. In addition, we are grateful to the orthopaedic doctors and nurses for their assistance and cooperation in patient management, operation implementation and data collection. Unfortunately, this list is not exhaustive, and we apologise for having been unable to acknowledge everyone.

\section{Abbreviations}

PVP: Percutaneous vertebroplasty; PKP: Percutaneous kyphoplasty; OVCFs: Osteoporotic vertebral compression fractures; IVC: Intravertebral vacuum cleft; ODI: Oswestry disability index; VAS: Visual analogue scale. IVP: Intravertebral vacuum phenomenon; BMD: Bone mineral density

\section{References}

[1] Lu W, Wang L, Xie C, et al. Analysis of percutaneous kyphoplasty or short-segmental fixation combined with vertebroplasty in the treatment of Kummell disease. J Orthop Surg Res. 2019; 14(1): 311.

[2] Jiang J, Gu F L, Li Z W, et al. The clinical efficacy and experience of bipedicular percutaneous vertebroplasty combined with postural reduction in the treatment of Kümmell's disease. BMC Musculoskelet Disord. 2020; 21(1): 82.

[3] Park J W, Park J H, Jeon H J, et al. Kümmell's Disease Treated with Percutaneous Vertebroplasty: Minimum 1 Year Follow-Up. Korean J Neurotrauma. 2017; 13(2): 119-23.

[4] Maldague B E, Noel H M, Malghem J J. The intravertebral vacuum cleft: a sign of ischemic vertebral collapse. Radiology. 1978; 129(1): 23-9.

[5] Feng S-W, Chang M-C, Wu H-T, et al. Are intravertebral vacuum phenomena benign lesions?. European spine journal : official publication of the European Spine Society, the European Spinal Deformity Society, 
and the European Section of the Cervical Spine Research Society. 2011; 20(8): 1341-8.

[6] Zhong W, Liang X, Luo X, et al. Vertebroplasty and vertebroplasty in combination with intermediate bilateral pedicle screw fixation for OF4 in osteoporotic vertebral compression fractures: a retrospective single-Centre cohort study. BMC Surg. 2019; 19(1): 178.

[7] Li H, Liang C Z, Chen Q X. Kümmell's disease, an uncommon and complicated spinal disorder: a review. The Journal of international medical research. 2012; 40(2): 406-14.

[8] Yu W, Liang D, Yao Z, et al. The therapeutic effect of intravertebral vacuum cleft with osteoporotic vertebral compression fractures: A systematic review and meta-analysis. International journal of surgery (London, England). 2017; 40: 17-23.

[9] Khan M, Kushchayev S V. Percutaneous Vertebral Body Augmentations: The State of Art. Neuroimaging clinics of North America. 2019; 29(4): 495-513.

[10] Zhang Y, Shi L, Tang P, et al. Comparison of the Efficacy Between Two Micro-Operative Therapies of Old Patients With Osteoporotic Vertebral Compression Fracture: A Network Meta-Analysis. Journal of cellular biochemistry. 2017; 118(10): 3205-12.

[11] Chang J Z, Bei M J, Shu D P, et al. Comparison of the clinical outcomes of percutaneous vertebroplasty vs. kyphoplasty for the treatment of osteoporotic Kummell's disease:a prospective cohort study. BMC Musculoskelet Disord. 2020; 21(1): 238.

[12] Liu F, Chen Z, Lou C, et al. Anterior reconstruction versus posterior osteotomy in treating Kümmell's disease with neurological deficits: A systematic review. Acta orthopaedica et traumatologica turcica. 2018; 52(4): 283-8.

[13] Huang Y-S, Hao D-J, Wang X-D, et al. Long-Segment or Bone Cement-Augmented Short-Segment Fixation for Kummell Disease with Neurologic Deficits? A Comparative Cohort Study. World neurosurgery. 2018; 116: e1079-86.

[14] Li Z, Liu T, Yin P, et al. The therapeutic effects of percutaneous kyphoplasty on osteoporotic vertebral compression fractures with or without intravertebral cleft. International orthopaedics. 2019; 43(2): 359-65.

[15] Lou S, Shi X, Zhang X, et al. Percutaneous vertebroplasty versus non-operative treatment for osteoporotic vertebral compression fractures: a meta-analysis of randomized controlled trials. Osteoporos Int. 2019; 30(12): 2369-80.

[16] Jiang J, Gu F-L, Li Z-W, et al. The clinical efficacy and experience of bipedicular percutaneous vertebroplasty combined with postural reduction in the treatment of Kümmell's disease. BMC musculoskeletal disorders. 2020; 21(1): 82. 
[17] Huang Y, Peng M, He S, et al. Clinical Efficacy of Percutaneous Kyphoplasty at the Hyperextension Position for the Treatment of Osteoporotic Kümmell Disease. Clinical spine surgery. 2016; 29(4): 161-6.

[18] Kim P, Kim S W. Balloon Kyphoplasty: An Effective Treatment for Kummell Disease?. Korean Journal of Spine. 2016; 13(3): 102-6.

[19] Li H-K, Hao D-J, Yang J-S, et al. Percutaneous kyphoplasty versus posterior spinal fixation with vertebroplasty for treatment of Kümmell disease: A case-control study with minimal 2-year follow-up. Medicine. 2017; 96(51): e9287.

[20] Park J W, Park J-H, Jeon H J, et al. Kümmell's Disease Treated with Percutaneous Vertebroplasty: Minimum 1 Year Follow-Up. Korean journal of neurotrauma. 2017; 13(2): 119-23.

[21] Sun $Y$, Xiong $X$, Wan D, et al. Effectiveness comparison between unilateral and bilateral percutaneous kyphoplasty for Kümmell disease. Zhongguo xiu fu chong jian wai ke za zhi = Zhongguo xiufu chongjian waike zazhi $=$ Chinese journal of reparative and reconstructive surgery. 2017; 31(9): 1086-91.

[22] Xia Y-H, Chen F, Zhang L, et al. Percutaneous kyphoplasty treatment evaluation for patients with Kümmell disease based on a two-year follow-up. Experimental and therapeutic medicine. 2018; 16(4): 3617-22.

[23] Zhang J, Fan Y, He X, et al. Is percutaneous kyphoplasty the better choice for minimally invasive treatment of neurologically intact osteoporotic Kümmell's disease? A comparison of two minimally invasive procedures. International orthopaedics. 2018; 42(6): 1321-6.

[24] Xiong X-M, Sun Y-L, Song S-M, et al. Efficacy of unilateral transverse process-pedicle and bilateral puncture techniques in percutaneous kyphoplasty for Kummell disease. Experimental and therapeutic medicine. 2019; 18(5): 3615-21.

[25] Ha K Y, Lee J S, Kim K W, et al. Percutaneous vertebroplasty for vertebral compression fractures with and without intravertebral clefts. The Journal of bone and joint surgery. British volume. 2006; 88(5): 62933.

[26] Molloy S, Mathis J M, Belkoff S M. The effect of vertebral body percentage fill on mechanical behavior during percutaneous vertebroplasty. Spine. 2003; 28(14): 1549-54.

[27] Graham J, Ahn C, Hai N, et al. Effect of bone density on vertebral strength and stiffness after percutaneous vertebroplasty. Spine. 2007; 32(18): E505-11.

[28] Hur W, Choi S S, Lee M, et al. Spontaneous Vertebral Reduction during the Procedure of Kyphoplasty in a Patient with Kummell's Disease. The Korean journal of pain. 2011; 24(4): 231-4.

[29] Hur W, Lee J J, Kim J, et al. Spontaneous air reduction of vertebra plana with Kummell's disease during vertebroplasty: subsequent experience with an intentional trial. Pain medicine (Malden, Mass.). 
[30] Patel A, Carter K R: Percutaneous Vertebroplasty And Kyphoplasty, StatPearls, Treasure Island (FL): StatPearls Publishing.2020.

[31] Huang S, Zhu X, Xiao D, et al. Therapeutic effect of percutaneous kyphoplasty combined with antiosteoporosis drug on postmenopausal women with osteoporotic vertebral compression fracture and analysis of postoperative bone cement leakage risk factors: a retrospective cohort study. Journal of orthopaedic surgery and research. 2019; 14(1): 452.

[32] Niu J, Zhou H, Meng Q, et al. Factors affecting recompression of augmented vertebrae after successful percutaneous balloon kyphoplasty: a retrospective analysis. Acta radiologica (Stockholm, Sweden : 1987). 2015; 56(11): 1380-7.

[33] Kim Y-Y, Rhyu K-W. Recompression of vertebral body after balloon kyphoplasty for osteoporotic vertebral compression fracture. European spine journal : official publication of the European Spine Society, the European Spinal Deformity Society, and the European Section of the Cervical Spine Research Society. 2010; 19(11): 1907-12.

[34] Chen W, Xie W, Xiao Z, et al. Incidence of Cement Leakage Between Unilateral and Bilateral Percutaneous Vertebral Augmentation for Osteoporotic Vertebral Compression Fractures: A Meta-Analysis of Randomized Controlled Trials. World neurosurgery. 2019; 122: 342-8.

[35] Zhan Y, Jiang J, Liao H, et al. Risk Factors for Cement Leakage After Vertebroplasty or Kyphoplasty: A Meta-Analysis of Published Evidence. World neurosurgery. 2017; 101: 633-42.

[36] Krauss $M$, Hirschfelder $H$, Tomandl $B$, et al. Kyphosis reduction and the rate of cement leaks after vertebroplasty of intravertebral clefts. European radiology. 2006; 16(5): 1015-21.

[37] Hu K Z, Chen S C, Xu L. Comparison of percutaneous balloon dilation kyphoplasty and percutaneous vertebroplasty in treatment for thoracolumbar vertebral compression fractures. Eur Rev Med Pharmacol Sci. 2018; 22(1 Suppl): 96-102.

[38] Yang C-C, Chien J-T, Tsai T-Y, et al. Earlier Vertebroplasty for Osteoporotic Thoracolumbar Compression Fracture May Minimize the Subsequent Development of Adjacent Fractures: A Retrospective Study. Pain physician. 2018; 21(5): E483-91.

\section{Tables}

Table 1 Baseline data of the two groups 


\begin{tabular}{lllll}
\hline Parameters & K group & O group & t/c & $P$ \\
\hline Cases & 35 & 35 & 0.396 & 0.567 \\
Gender & 11 & 9 & & \\
$\quad$ Male (cases) & 24 & 26 & & \\
$\quad$ Female (cases) & $76.0 \pm 5.8$ & $75.5 \pm 5.9$ & -0.532 & 0.433 \\
Age (years) & $8.4 \pm 3.0$ & $0.28 \pm 0.17$ & -15.960 & 0.000 \\
Course of disease (months) & & & 11.209 & 0.001 \\
Fall history & 11 & 25 & & \\
$\quad$ Yes(cases) & 24 & 10 & 0.484 & 0.922 \\
$\quad$ No (cases) & 7 & 5 & & \\
Injured vertebral segment & 12 & 13 & & \\
$\quad$ T11(cases) & 12 & 12 & & \\
$\quad$ T12(cases) & 4 & 5 & -1.124 & 0.037 \\
$\quad$ L1(cases) & $-4.25 \pm 0.91$ & $-3.88 \pm 0.81$ & -1.5 \\
$\quad$ L2(cases) & $36.9 \pm 8.79$ & $31.5 \pm 8.46$ & 2.447 & 0.018 \\
BMD (T value) & & & & \\
Vertebral compression rate $[\% \square$ & & & & \\
\hline
\end{tabular}

Table 2 Comparison of clinical outcomes between the two groups

\begin{tabular}{|c|c|c|c|c|}
\hline Parameters & K group & O group & $t / c^{2}$ & $P$ \\
\hline Follow-up time(months) & $35.4 \pm 6.89$ & $35.9 \pm 7.53$ & 0.215 & 0.919 \\
\hline Amount of bone cement injected $\square \mathrm{ml} \square$ & $4.2 \pm 1.65$ & $3.6 \pm 1.35$ & 1.875 & 0.025 \\
\hline Operation time ( $\mathrm{min})$ & $36.8 \pm 3.57$ & $35.1 \pm 5.15$ & 0.132 & 0.696 \\
\hline Intraoperative blood loss (ml) & $16.9 \pm 3.45$ & $16.2 \pm 3.28$ & 5.479 & 0.509 \\
\hline Fluoroscopy times & $16.7 \pm 3.50$ & $15.9 \pm 3.46$ & 4.060 & 0.453 \\
\hline \multicolumn{3}{|l|}{ Bone cement distribution } & 11.283 & 0.001 \\
\hline local solid lump distribution & 23 & 9 & & \\
\hline Nested distributions & 12 & 26 & & \\
\hline \multicolumn{3}{|l|}{ Bone cement leakage } & 0.565 & 0.452 \\
\hline Yes (cases) & 5 & 3 & & \\
\hline No (cases) & 30 & 32 & & \\
\hline \multicolumn{5}{|l|}{ VAS scores } \\
\hline Before surgery & $8.0 \pm 0.77$ & $7.8 \pm 0.75$ & 0.458 & 0.186 \\
\hline At 1 day after surgery & $2.8 \pm 0.73 *$ & $1.7 \pm 0.81 *$ & 1.301 & 0.013 \\
\hline At 1 year after surgery & $2.4 \pm 0.68 *$ & $1.6 \pm 0.84 *$ & 1.398 & 0.025 \\
\hline At 2 years after surgery & $2.5 \pm 0.70^{*}$ & $1.5 \pm 0.84^{*}$ & 1.356 & 0.022 \\
\hline \multicolumn{5}{|l|}{ ODI } \\
\hline Before surgery & $84.1 \pm 7.89$ & $84.6 \pm 7.73$ & -2.293 & 0.662 \\
\hline At 1 day after surgery & $29.4 \pm 5.42 *$ & $25.4 \pm 5.13 *$ & 3.533 & 0.026 \\
\hline At 1 year after surgery & $29.8 \pm 5.39 *$ & $24.9 \pm 5.01 *$ & 3.407 & 0.024 \\
\hline At 2 years after surgery & $29.9 \pm 5.41 *$ & $25.0 \pm 5.56 *$ & 3.872 & 0.034 \\
\hline \multicolumn{3}{|l|}{ Adjacent vertebral fractures } & 0.255 & 0.243 \\
\hline Yes (cases) & 6 & 4 & & \\
\hline No (cases) & 29 & 31 & & \\
\hline
\end{tabular}

* Compared to before surgery, $P=0.000$

Table 3 Comparison of the two groups of imaging data

\begin{tabular}{lllll}
\hline Parameters & K group & O group & t/c & $P$ \\
\hline Correction rate of vertebral compression & & & & \\
At 1 day after operation & $20.9 \pm 5.54$ & $13.8 \pm 4.29$ & 6.515 & 0.000 \\
At 1 year after operation & $17.8 \pm 5.33^{*}$ & $12.5 \pm 3.79$ & 5.248 & 0.000 \\
At 2 years after operation & $16.7 \pm 5.15^{*}$ & $12.1 \pm 3.61^{*}$ & 5.290 & 0.001 \\
Correction rate of kyphosis & & & & \\
At 1 day after operation & $21.0 \pm 5.34$ & $14.6 \pm 5.69$ & 5.467 & 0.000 \\
At 1 year after operation & $17.9 \pm 5.24^{*}$ & $13.9 \pm 5.26$ & 6.093 & 0.001 \\
At 2 years after operation & $17.2 \pm 5.17^{*}$ & $13.3 \pm 5.19^{*}$ & 4.509 & 0.001 \\
\hline
\end{tabular}

* Compared with 1 day after operation, $P<0.05$ 
Figures

Cases of Kümmell 's disease and OVCFs

Inclusion criteria

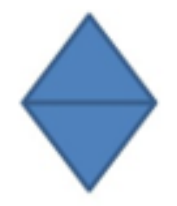

Exclusion criteria

A total 70 cases of Kümmell 's disease and OVCFs treated with PVP (February 2016-February 2018)

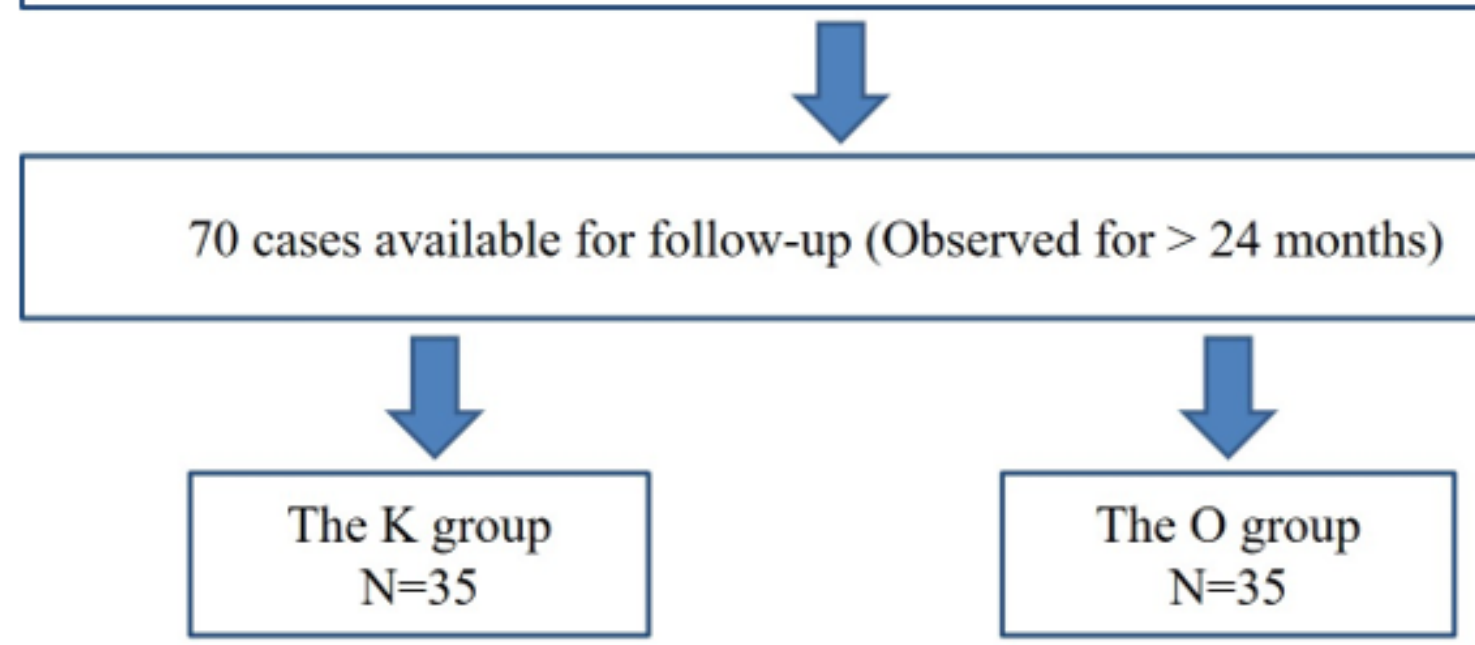

Figure 1

A schematic diagram of case grouping and follow-up process 

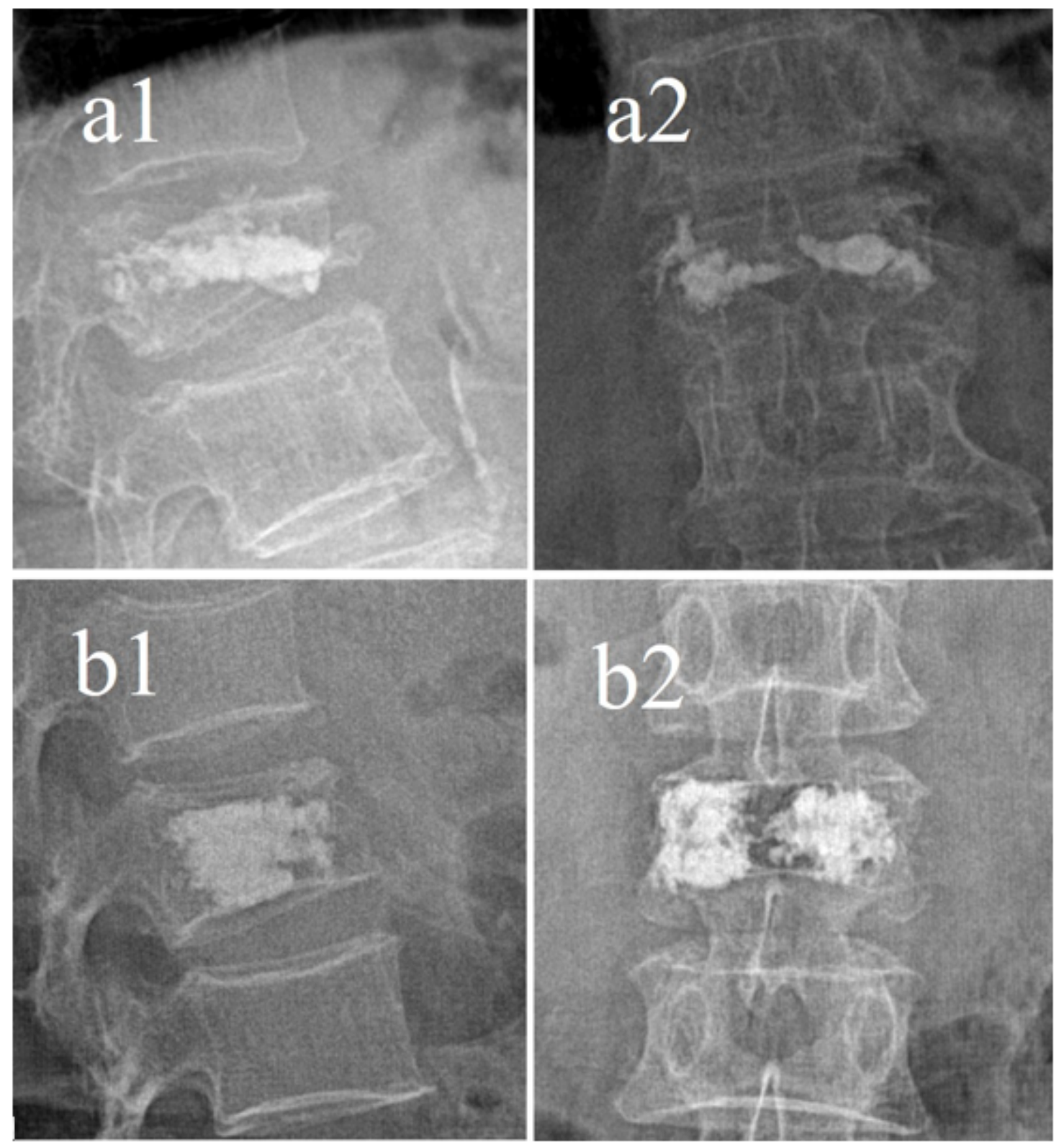

Figure 2

Distribution pattern of bone cement: a local solid lump of distribution pattern; $b$ embedded trabeculae of distribution pattern 